\title{
What Is Interventional Radiology?
}

\author{
Sun H. Abn, MD, and Mabesh V. Jayaraman, MD
}

Coined by Dr. Alexander Margulis in 1967, interventional radiology is a medical specialty devoted to patients' clinical care in an image-guided, innovative, and minimally invasive manner. Dr. Charles Dotter first introduced interventional radiology to the world in 1964, when he percutaneously dilated a superficial femoral artery stenosis in an 82 year-old woman and averted an amputation. Since then, interventional radiologists have pioneered treatments in many areas of medicine, including balloon angioplasty and stenting in peripheral vascular disease, catheter directed thrombolysis, embolization for cessation of bleeding, needle biopsies and drainages, etc. Many recent and exciting advances have come in the areas of peripheral vascular disease, oncology, uterine fibroid disease, varicose vein management, and dialysis and venous access. In neurointerventional radiology, endovascular therapies for intracranial aneurysms, vascular malformations and atherosclerotic occlusive disease have expanded treatment options for many patients.

Perhaps more important than the technical advances, the field has evolved from a procedure-oriented specialty to a clinical practice with commitment to direct patient care. Interventional radiologists routinely perform inpatient and outpatient consultations, make diagnoses, perform treatments, and longitudinally follow their patients. This issue highlights some of the latest advances in the field of interventional and neurointerventional radiology. For more information please refer to Society of Interventional Radiology (www.sirweb.org) and Society of NeuroInterventional Surgery (www.snisonline.org).
Sun H. Ahn, MD, is the Director of the Vascular and Interventional Radiology Fellowship Program at Rhode Island Hospital and an Assistant Professor (Clinical) of Diagnostic Imaging.

Mahesh V. Jayaraman, MD, is Assistant Professor of Diagnostic Imaging and Neurosurgery.

\section{Disclosure of Financial Interests \\ The authors have no financial inter- ests to disclose.}

\section{Correspondence}

Sun H. Ahn, MD

Department of Diagnostic Imaging

Rhode Island Hospital

593 Eddy St.

Providence, RI 02903

phone: (401) 444-5194

e-mail:sahn@lifespan.org

\section{Intracranial Aneurysms: Perspectives On the Disease and Endovascular Therapy}

Awais Z. Vance, MD, Mahesh V. Jayaraman, MD, Richard A. Haas, MD, Curtis E. Doberstein, MD

There are approximately 30,000 cases per year in the United States of aneurysmal subarachnoid hemorrhage (SAH), leaving $60 \%$ of patients dead or disabled. However, improvements in treatment have occurred in the past few decades; a recent meta-analysis shows decreasing case fatality rates from 1973 to $2002 .{ }^{1}$

This article will discuss the epidemiology, natural history, diagnosis and treatment of intracranial aneurysms. We hope that the reader will leave with an understanding of the strengths and limitations of endovascular treatment for intracranial aneurysms.

\section{Epidemiology and Natural History}

Intracranial aneurysms are present in $1-5 \%$ of the general population; ${ }^{2} 10$ to $30 \%$ of patients will have multiple aneurysms. ${ }^{3}$ The majority of aneurysms occur in the anterior circulation near the Circle of
Willis with the most common locations in descending frequency being the anterior communicating artery (Acomm), posterior communicating artery (Pcomm), and middle cerebral artery (MCA).

Ruptured aneurysms most often present with subarachnoid hemorrhage. After the initial hemorrhage, the highest risk for rehemorrhage occurs in the first 2 weeks at a rate of $1-2 \%$ / day. If untreated, $50 \%$ of patients will re-hemorrhage within 6 months of initial hemorrhage; up to $72 \%$ of these patients will suffer death or severe disability. 4,5 While historically, patients were treated 2 to 3 weeks after hemorrhage, the International Cooperative study on timing of Aneurysm surgery ${ }^{6,7}$ showed that early treatment was superior. The current standard of care is to treat ruptured intracranial aneurysms as soon as reasonably possible after hemorrhage. Upon presentation, patients are assessed clinically using the Hunt and Hess grading system, from I (minimal headache, no other symptoms) to $\mathrm{V}$ (comatose, moribund). As would be expected, the lower the grade at presentation, the better the outcome.

\section{RUPTURE RisK OF UnRUPTURED ANEURYSMS}

Unruptured aneurysms may present with headache, stroke, neurologic deficits related to mass effect or as incidental findings on imaging. The International Study of Unruptured Intracranial Aneurysms (ISUIA) ${ }^{8}$ showed that the risk of rupture was related to the size, location and history of prior SAH. The 5year rupture rates are summarized in Table 1. To summarize their findings, larger aneurysms, those in the posterior circulation (or Posterior communicating artery), and those in patients with prior history of SAH are all associated with higher rupture rates. In general, most small $(<7 \mathrm{~mm})$ aneurysms in the anterior 
circulation in a patient with no prior personal or family history of SAH can be followed with serial imaging.

Patients with a family history of aneurysm are also at higher risk for rupture for small aneurysms. ' While these prospective rupture rates are helpful, the ultimate decision to treat or observe an unruptured aneurysm depends on several other factors, including the patient's age, medical status, family history and personal preferences.

\section{Imaging Evaluation}

The most appropriate imaging study for evaluation of intracranial aneurysms depends on the clinical presentation. For patients with subarachnoid hemorrhage, the most sensitive examination is required because even very small aneurysms are important to detect. For unruptured aneurysms, a safe (though perhaps less sensitive) imaging modality is preferred because very small aneurysms $(<3 \mathrm{~mm})$ are unlikely to make a clinical difference. The available imaging modalities include MR angiography (MRA), CT angiography (CTA) and catheter-based digital subtraction angiography (DSA).

MRA is the least sensitive modality, especially for aneurysms $<3 \mathrm{~mm}$ in size, but uses no ionizing radiation or intravenous contrast. The sensitivity of MRA varies with aneurysm size, with MRA being best for aneuryms $5 \mathrm{~mm}$ or larger. CTA is more sen- sitive than MRA, especially for aneurysms $<5 \mathrm{~mm}$ in size. However, the patient is subjected to the risks of ionizing radiation and iodinated contrast. In aneurysms greater than $5 \mathrm{~mm}$ in size, the sensitivity for detection is equal to MRA though CTA is superior in characterization of vascular anatomy of adjacent branches. ${ }^{10,11,12,13,14}$

Angiography is the most sensitive examination for intracranial aneurysms. It is minimally invasive with a low rate of permanent neurologic morbidity. Recent studies have shown that permanent complication rates at busy neurovascular centers are extremely low, ${ }^{15}$ typically much less than $0.1 \%$. Angiography's spatial resolution exceeds that of CTA and MRA by almost an order of magnitude. Newer 3DDSA technology allows for detailed reconstructions that can allow for examining the aneurysm from a variety of angles and facilitates treatment planning.
For patients previously treated with surgical clips, both MRA and CTA have artifact that precludes adequate evaluation of the treated aneurysm. In those cases, angiography is often necessary. For patients treated with endovascular coils, MRA is an ideal way to follow treated aneurysms, as there is little artifact caused from the coils.

A common question is: who should be screened for aneurysms? The current recommendations are to screen patients in whom at least two immediate relatives have a documented intracranial aneurysm, and all patients with adult polycystic kidney disease. $^{3,16,17}$ Familial aneurysms represent approximately $15 \%$ of all aneurysm patients.

In modern practice, MRA is typically used for screening of asymptomatic high risk patients and for follow up of previously detected or previously coiled aneurysms. CTA is often used to characterize a questionable MRA abnormality and oc-
TABLE 1: Prospective 5-year cumulative rupture rates from the International study of unruptured intracranial aneurysms (ISUIA)

\begin{tabular}{|c|c|c|c|c|c|}
\hline \multirow[t]{2}{*}{ Aneurysm Size } & $<7 \mathrm{~mm}$ & & $7-12 \mathrm{~mm}$ & $13-24 \mathrm{~mm}$ & $>25 \mathrm{~mm}$ \\
\hline & $\begin{array}{l}\text { Group I } \\
\text { (no prior } \\
\text { SAH) }\end{array}$ & $\begin{array}{l}\text { Group II } \\
\text { (prior } \\
\text { SAH) }\end{array}$ & & & \\
\hline Cavernous ICA & 0 & 0 & 0 & $3.0 \%$ & $6.4 \%$ \\
\hline Anterior Circulation & 0 & $1.5 \%$ & $2.6 \%$ & $14.5 \%$ & $40 \%$ \\
\hline $\begin{array}{l}\text { Posterior Circulation } \\
\text { (incl. PComm) }\end{array}$ & $2.5 \%$ & $3.4 \%$ & $14.5 \%$ & $18.4 \%$ & $50 \%$ \\
\hline
\end{tabular}

\begin{tabular}{|c|c|c|c|}
\hline \multicolumn{4}{|c|}{$\begin{array}{c}\text { TABLE 2: Typical indications, advantages and disadvantages for available } \\
\text { aneurysm imaging modalities: }\end{array}$} \\
\hline Modality & Indications & Strengths & Weaknesses \\
\hline MRA & $\begin{array}{l}\text { - Screening of high risk } \\
\text { asymptomatic patients } \\
\text { - Follow up of previously } \\
\text { detected aneurysms } \\
\text { - Follow up of previously } \\
\text { coiled aneurysms }\end{array}$ & $\begin{array}{l}\text { - No ionizing radiation } \\
\text { - No intravenous } \\
\text { contrast required } \\
\text { - Good for coiled } \\
\text { aneurysms }\end{array}$ & $\begin{array}{l}\text { - Limited sensitivity for } \\
<5 \mathrm{~mm} \text { aneurysms } \\
\text { - Substantial artifact for } \\
\text { clipped aneurysms }\end{array}$ \\
\hline CTA & $\begin{array}{l}\text { - Characterize a } \\
\text { questionable MRA } \\
\text { abnormality } \\
\text { - Occasionally for follow up } \\
\text { of aneurysms difficult to } \\
\text { visualize by MRA }\end{array}$ & $\begin{array}{l}\text { - Better spatial resolution } \\
\text { then MRA } \\
\text { - Least time consuming }\end{array}$ & $\begin{array}{l}\text { - Ionizing radiation required } \\
\text { - Iodinated contrast required } \\
\text { - Less sensitive then DSA } \\
\text { for }<3 \mathrm{~mm} \text { aneurysms }\end{array}$ \\
\hline DSA & $\begin{array}{l}\text { - Suspected aneurysmal SAH } \\
\text { - Treatment planning when } \\
\text { definitive characterization } \\
\text { and anatomic definitions } \\
\text { necessary }\end{array}$ & $\begin{array}{l}\text { - Highest spatial resolution } \\
\text { - Can image and treat in the } \\
\text { same setting }\end{array}$ & $\begin{array}{l}\text { - Minimally Invasive } \\
\text { - lonizing radiation required } \\
\text { - lodinated contrast required }\end{array}$ \\
\hline
\end{tabular}



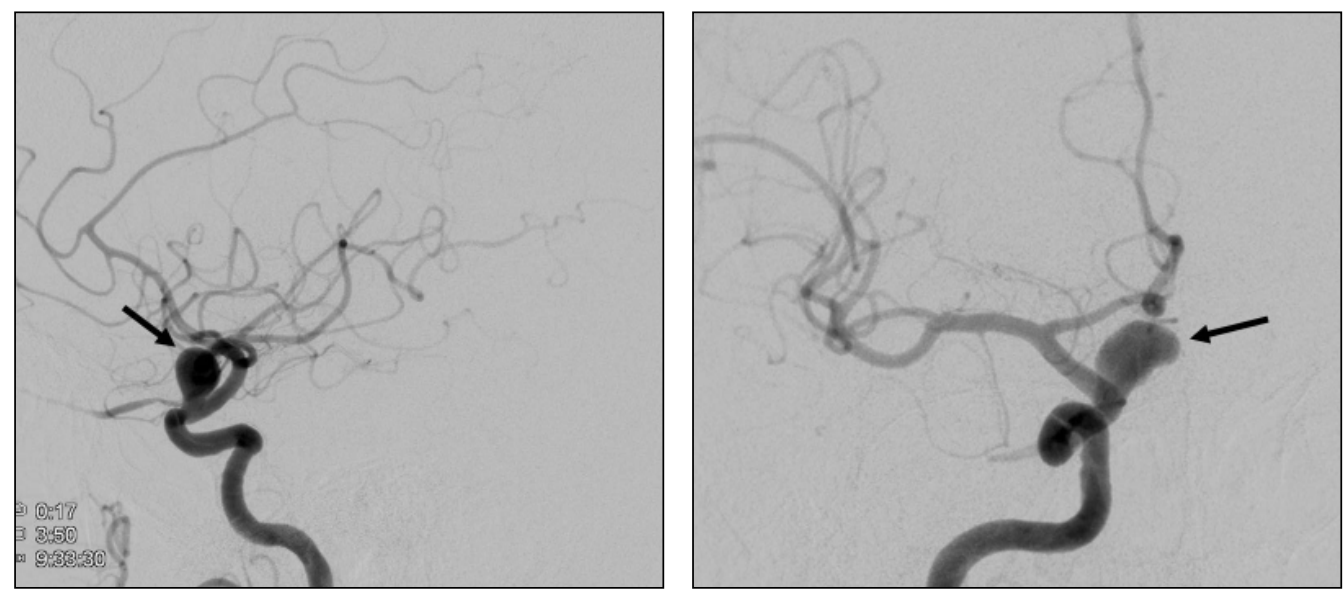

Figure 1. Angiogram images from a 62 year-old male with an unruptured aneurysm. Lateral (upper left) and Frontal (upper right) angiogram images demonstrate a $12 \mathrm{~mm}$ aneurysm (arrows) arising from the internal carotid artery.

Images after endovascular therapy show coils (arrow, lower left) in the aneurysm. Angiogram following coiling shows no residual filling of the aneurysm (lower right).
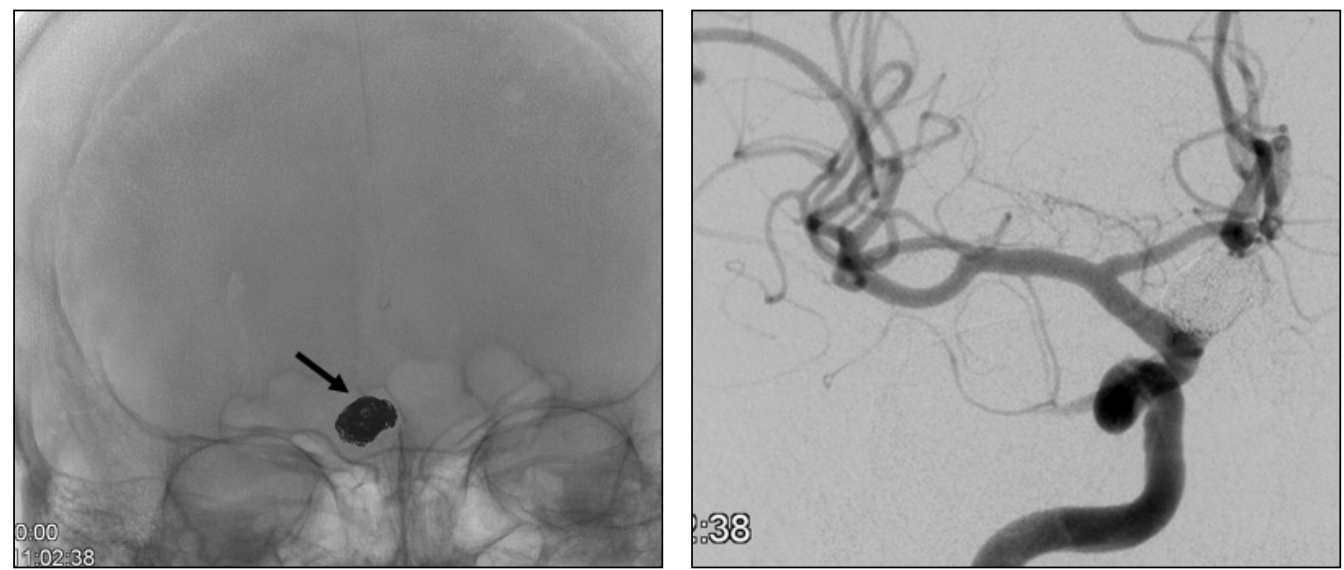

casionally for follow up of aneurysms which are difficult to visualize by MRA. DSA is the gold standard and should be performed on all SAH patients since it is important to detect even the smallest of aneurysms in this population. DSA is also used in treatment planning when definitive characterization and anatomic definition is needed. These imaging recommendations are summarized in Table 2.

\section{Treatment Options}

The goal of aneurysm treatment is to prevent hemorrhage or re-hemorrhage. Treatment should be safe, effective and durable. The two main options are craniotomy with microsurgical clipping or endovascular coiling. Although neurosurgical clipping is invasive, it has been the traditional treatment with a long track record, with the major advantage of durability. Re-hemorrhage and recurrence rates of surgically clipped aneurysms are extremely low, and routine imaging follow up is typically not necessary.

Endovascular therapy for aneurysms has been performed for several decades, but the Guglielmi Detachable Coil (GDC, Boston Scientific, Natick, MA) in the early 1990s revolutionized the field. In this procedure, a very small microcatheter is placed into the aneurysm sac, and progressively smaller coils are placed to induce thrombosis of the aneurysm, all through a small femoral arterial access. Initially, coil embolization was limited to aneurysms with a narrow opening (neck) to the aneurysm to facilitate coil placement. However, newer balloon and stent-assisted techniques have greatly expanded endovascular treatment horizons. When using balloon-assisted technique, a balloon is inflated at the base of the aneurysm in order to prevent coils from protruding out of the aneurysm and into the parent vessel. For stent-assisted coiling, newer endovascular stents specifically designed for this purpose have been designed. (Figures 1 and 2) One drawback of stents is that the required anti-platelet agents can pose a potential problem in acutely ruptured aneurysms.

\section{Comparative Studies of Clipping and Coiling}

When the GDC coil was first introduced, the coiling procedure was typically reserved for poor Hunt \& Hess grade patients. Gradually, however, greater use of coiling was applied to patients with better clinical grades. The landmark International Subarachnoid Hemorrhage Trial (ISAT) enrolled patients from 1995 onward, mostly in Europe. Patients with good clinical grades who had aneurysms which could reasonably undergo either surgical or endovascular therapy were randomized. The initial data, published in 2003 (after 2143 patients had been randomized), showed a statistically significant reduction in percentage of patients dead or disabled at one year, from $30.9 \%$ (surgery) to $23.5 \%$ (coiling). Among the initial criticisms of the study were the lack of long term follow-up, and the fact that surgical experience among the European centers may not be comparable to those of major US medical centers. However, more recent longterm data have shown that the coiling treatment is indeed durable, with a statistically lower risk of death at 5 years when compared with clipping. ${ }^{18}$ While the risk of rehemorrhage from a clipped aneurysm was significantly lower than that of a coiled aneurysm, the rates of both were low and did not overwhelm the early benefit of the less invasive procedure. ISAT also established that the rate of recurrent $\mathrm{SAH}$ in these pa- 
tients was both from treated aneurysms but also from other aneurysms, either those present initially or those which formed in the interim. This stresses the need for longterm follow-up of patients with prior SAH, irrespective of whether they were treated with coiling or clipping. While these results from a landmark study cannot be generalized to every patient, they suggest that if a patient has subarachnoid hemorrhage and an aneurysm that can be treated with either modality, then coiling is associated with a lower rate of death or dependency. A randomized trial at a high volume US neurosurgical center (Barrow Neuroscience Institute, Phoenix, AZ) confirmed these findings, showing a statistically significant benefit to coiling over clipping in patients who could undergo both treatments. ${ }^{19}$

\section{...the current standard of care is to treat ruptured intracranial \\ aneurysms as soon as reasonably possible after hemorrhage.}

Unlike ruptured aneurysms, no randomized trial compares surgical and endovascular treatment for unruptured aneurysms. With unruptured aneurysms, the surgical limitations of operating on an acutely injured brain are not present as they are in the setting of SAH, and the goal should be to attempt to give the patient a durable treatment for their aneurysm. The ISUIA study, comparing the outcomes of surgical clipping with endovascular coiling, found that the 1year morbidity/mortality for endovascular coiling $(9.8 \% / 7.1 \%)$ was lower than that of surgical clipping (12.6\%/10.1\%). They study found that poorer surgical outcome was associated with age greater than 50, larger aneurysms, posterior circulation location and in patients with history of ischemic cerebrovascular disease. Poorer prognosis associated with endovascular treatment was less dependent on patient age and aneurysm size though aneurysms $>12 \mathrm{~mm}$ and those lo-
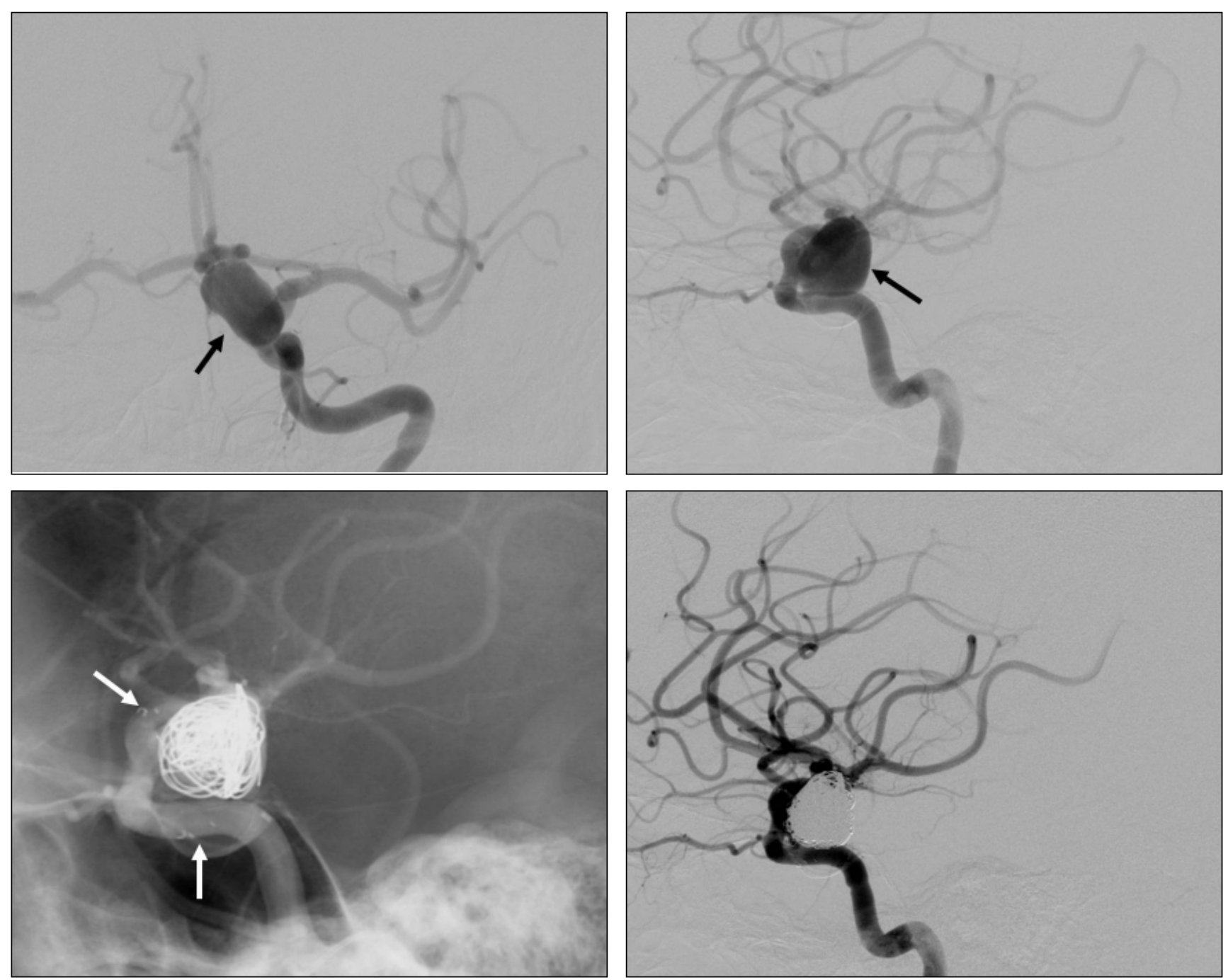

Figure 2. Stent-assisted endovascular therapy of a large aneurysm arising from the left internal carotid artery. Frontal (upper left) and Lateral (upper right) angiograms show the aneurysm (arrows). Note the relatively wide neck (opening) into the aneurysm, as compared with the narrow neck in Figure 1. In this case, treatment was performed using a stent-assisted technique. Post-coiling images (lower left, lower right) show the coils within the aneurysm, without obstructing parent vessel flow. Note the markers indicating the location of the stent within the internal carotid artery (arrows, lower left). Post-treatment angiogram shows patent flow through the parent vessel with only minimal filling of the aneurysm (lower right). 

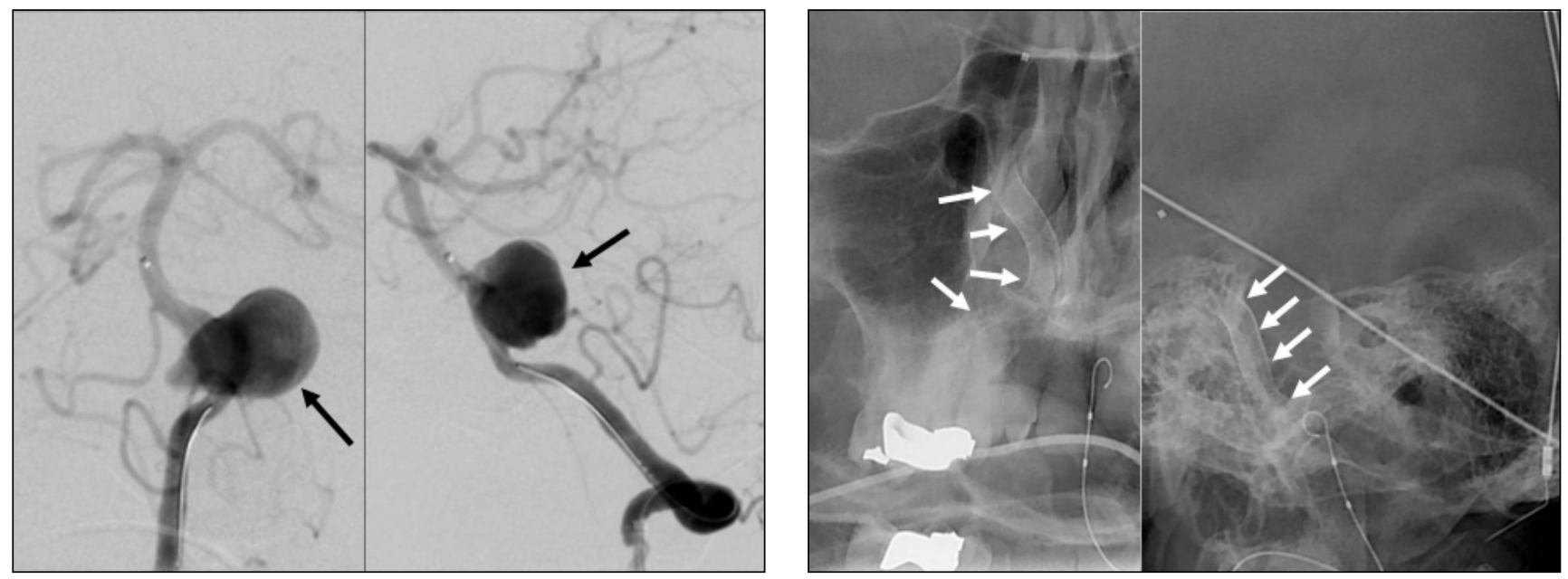

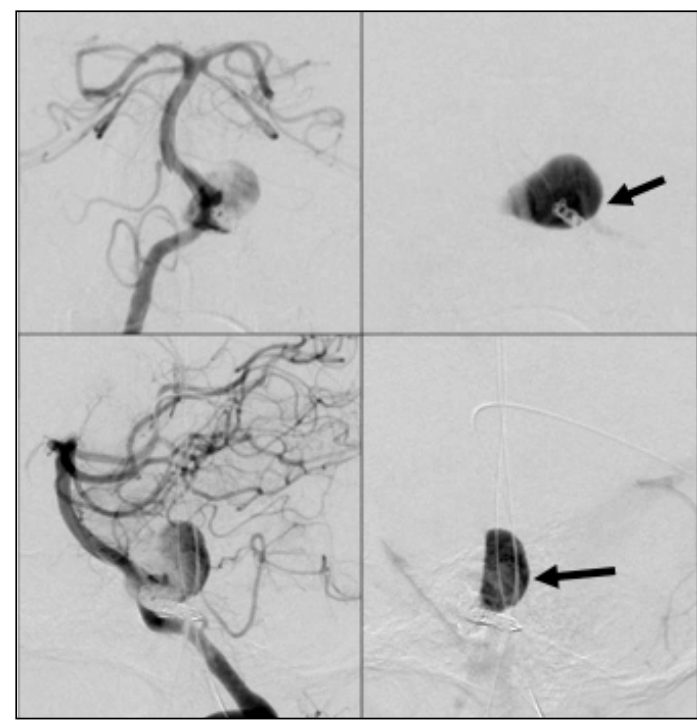

cated in the posterior circulation portended a poorer prognosis. ${ }^{8}$ Higashida et al performed a retrospective analysis of discharge results in 18 states of $2619 \mathrm{pa}-$ tients treated for unruptured aneurysms at 429 community and academic hospitals. They found that endovascular treatment of unruptured aneurysms was associated with statistically significant reductions in morbidity, mortality and decreased hospital use at discharge. ${ }^{20}$ However, both these studies were not randomized, and the data should be interpreted as such, acknowledging the substantial limitation of patient selection bias.

\section{Endovascular Therapy: LIMITATIONS AND FUTURE Directions}

The greatest limitations of endovascular therapy have been the durability of treatment for large and giant aneurysms, and therefore the implicit need for follow-up imaging. ISAT showed that
Figure 3. Treatment of a complex, large aneurysm at the vertebro-basilar junction using a flow diverting stent. Initial angiogram images prior to treatment (upper left) show a large aneurysm (arrows) at the confluence of both vertebral arteries to form the basilar artery. Conventional coil embolization or surgery for this lesion would be difficult. Native images (upper right) show a flow diverting stent (arrows) placed from the right vertebral artery to the basilar artery, in order to disrupt flow across the neck of this aneurysm and induce thrombosis. Final post treatment angiogram (lower left) shows stasis of contrast (arrows) well after contrast has washed out of the normal arteries and veins. It is expected this portion of the aneurysm will slowly thrombose and shrink down over time. endovascular therapy was indeed durable with respect to preventing re-hemorrhage, but that patient population was mostly small aneurysms. ${ }^{18}$ Murayama et al reported aneurysm recurrence rates post coiling from their experience at UCLA from 19912002. ${ }^{21}$ They found that progressively larger and wider neck aneurysms had progressively higher recurrence rates, ranging from 5.2\% for small aneurysms (with a small neck) to $63.2 \%$ for giant aneurysms. The mean time to recurrence following treatment was 12 months, with most recurrences

\section{The most exciting future development in endovascular aneurysm therapy is the flow diverting stent (FDS).}

by 36 months in a large series. ${ }^{22}$ They also found that the major risk factors for recurrence included aneurysm size $>10 \mathrm{~mm}$ and incomplete initial occlusion. Thus one of the limitations of endovascular treatment remains the durability in large and wide neck aneurysms.

The most exciting future development in endovascular aneurysm therapy is the flow diverting stent (FDS). Unlike stents used to assist in placing coils into an aneurysm, these devices are designed to be the only therapy used to treat an aneurysm. As such, they have a higher degree of metal coverage than stents currently used. The device is placed in the parent vessel harboring the aneurysm, and by doing so causes marked disruption of the flow vectors leading into the aneurysm. This induces thrombosis of the aneurysm, and animal studies have shown that eventually a neo-intima grows over the previous opening to the aneurysm. This concept is extremely promising, since it ad- 
dresses the issues of recurrence of large and giant aneurysm. While none of the devices are FDA-approved, studies outside of the US have shown great promise. The Pipeline Embolization Device (Ev3 neurovascular, Irvine, CA) is the best studied to date, with several hundred human cases performed. (Figure 3) Single center studies have shown very impressive results in treating the large and giant aneurysms endovascularly using that device. ${ }^{23} \mathrm{Com}$ plete thrombosis of the aneurysm can take weeks to months, and eventually the aneurysm sac collapses and restores a normal parent vessel contour. Remarkably, the small perforating arteries which arise from the vessel segment seem to stay patent. Most of the work using FDS has not involved bifurcation aneurysms, so their applicability in that situation is unknown. Much work remains to be done with these devices, and their use in acutely ruptured aneurysms would require the use of antiplatelet agents. All these issues would need to be addressed before they could supplant coils as the major therapy for intracranial aneurysms. If indeed further studies confirm the promising preliminary data, the landscape for aneurysm therapy will change greatly in the coming decade.

\section{What's Best For My Patient?}

One of the most common questions is: "what's better: coiling or clipping?" The answer is "it depends..." Certainly the patient's clinical condition and medical co-morbidities should be considered. Depending on their morphology and location, aneurysms may be better suited for one therapy over another. While the trend has been towards a greater percentage of aneurysms treated endovascularly, many aneurysms are still better suited for surgical therapy. For example, MCA aneurysms are often treated surgically owing to their combination of easier surgical exposure than other locations, and their tendency to have wide necks and branch vessel incorporation, anatomic factors which make endovascular treatment more difficult. ${ }^{24}$ For patients with an aneurysm that can be treated using either modality, a frank discussion with the patient of the benefits and drawbacks of both options is warranted. In our practice, we discuss the cases jointly whenever possible and frankly discuss the pros and cons of both therapies with our patients.

\section{Conclusion}

Intracranial aneurysms are present in $1-5 \%$ of the population, most often presenting with subarachnoid hemorrhage. For unruptured aneurysms, larger size (especially $>6 \mathrm{~mm}$ ), posterior circulation location, personal or family history of aneurysm rupture are all associated with higher prospective rates of hemorrhage. Treatment of intracranial aneurysms has evolved over the past two decades, with endovascular coiling offered as primary therapy in a greater percentage of patients. This is primarily based on the results of a large scale randomized trial showing improved outcomes with coiling over surgical clipping in patients with ruptured aneurysms suited for both therapies. Both clipping and coiling are effective at preventing hemorrhage and durable over the long term, with slightly lower rates of re-hemorrhage with clipping compared with coiling. Future endovascular directions may involve the use of flow diverting stents, which induce aneurysm thrombosis over time and show early promise in the most difficult of aneurysms. Aneurysms should be treated at a center that can offer both surgical clipping and coiling in order to result in the best possible patient outcomes.

\section{References}

1. Nieuwkamp DJ, Setz LE, et al. Lancet Neurol 2009;8:635-42.

2. Wiebers DO, Whisnant JP, et al. Lancet 2003;362:103-10.

3. Schievink WI. NEJM 1997;336:28-40.

4. Hurst RW, RH Rosenwasser. Interventional Neuroradiology. New York: Informa; 2008.

5. Greenberg MS. Handbook of Neurosurgery. New York: Thieme, 2006.

6. Kassell NF, Torner JC, et al. The International Cooperative Study on the Timing of Aneurysm Surgery Part 1. J Neurosurg 1990;73:1, 18-36.

7. Kassell NF, Torner JC, et al. J Neurosurg 1990;73: 37-47.

8. International Study of Unruptured Intracranial Aneurysms Investigators. Lancet 2003;362:10310.

9. Broderick JP, Brown RD, et al. Stroke 2009; 40:1952

10. White PM, Teadsale E, et al.J Neurol Neurosurg Psychiatry 2001;71:322-8.

11. McKinney AM, Palmer CS, et al. Amer J Neuroradiol 2008;29:594-602.

12. Lubicz B, Levivier M, et al. American J Neuroradiol 2007;28:1949-55.
13. Deutschmann HA, Augustin M, et al. Amer J Neuroradiol 2007;28:628-34.

14. Adams WM, Laitt RD, A Jackson. Amer J Neuroradiol 2000;21:1618-28.

15. Fifi JT, Meyers PM, et al. J Vascular Interventional Radiol 2009;20:442-7.

16. Butler WE, Barker FG II, Crowell RM. Neurosurg 1996;38:506-15.

17. The Magnetic Resonance Angiography in Relatives of Patients with Subarachnoid Hemorrhage Study Group. NEJM 1999;341:1344-50.

18. Molyneux AJ, Kerr R, et al. Lancet 2009; 8:42733.

19. McDougall CG. Barrow Ruptured Aneurysm Trial. American Association of Neurological Surgeons Annual Meeting, 20-22 Feb 2008. Chicago, IL 2008.

20. Higashida RT, Lahue BJ, et al. American J Neuroradiol 2007;28:146-51.

21. Murayama Y, Nien YL, et al. J Neurosurg 2003;98:959-66.

22. Raymond J, Guilbert F, et al. Stroke 2003;34:1398-403.

23. Lylyk P, Miranda C, et al. Neurosurg 2009;64:632-42

24. Jayaraman MV, Do HM, et al. JStroke Cerebrovascular Dis 2007;16:52-6.

Awais $Z$. Vance, $M D$, is in his final year of residency in the Department of Diagnostic Imaging and will be pursuing further training in Interventional Neuroradiology.

Mahesh V. Jayaraman, MD, is Assistant Professor of Diagnostic Imaging and Neurosurgery.

Richard A. Haas, MD, is Associate Professor (Clinical) of Diagnostic Imaging and Neurosurgery.

Curtis E. Doberstein, MD, is Associate Professor (Clinical) of Neurosurgery and Director of Cerebrovascular Neurosurgery. All are at the Warren Alpert Medical School of Brown University.

\section{Disclosure of Financial Interests}

The authors have no financial interests to disclose.

\section{Correspondence to:}

Mahesh V. Jayaraman, MD

Department of Diagnostic Imaging

Rhode Island Hospital

593 Eddy Street, 3rd Floor Main

Providence, RI 02903

Phone: (401) 444-5184

E-mail: MJayaraman@lifespan.org 